\title{
Strongly Interacting Matter Under Rotation: An Introduction
}

\author{
Francesco Becattini, Jinfeng Liao, and Michael Lisa
}

\begin{abstract}
Ultrarelativistic collisions between heavy nuclei briefly generate the quark-gluon plasma (QGP), a new state of matter characterized by deconfined partons last seen microseconds after the Big Bang. The properties of the QGP are of intense interest, and a large community has developed over several decades, to produce, measure and understand this primordial plasma. The plasma is now recognized to be a strongly-coupled fluid with remarkable properties, and hydrodynamics is commonly used to quantify and model the system. An important feature of any fluid is its vorticity, related to the local angular momentum density; however, this degree of freedom has received relatively little attention because no experimental signals of vorticity had been detected. Thanks to recent high-statistics datasets from experiments with precision tracking and complete kinemetic coverage at collider energies, hyperon spin polarization measurements have begun to uncover the vorticity of the QGP created at the Relativistic Heavy Ion Collider. The injection of this new degree of freedom into a relatively mature field of research represents an enormous opportunity to generate new insights into the physics of the QGP. The community has responded with enthusiasm, and this book (to be published as a volume of Lecture Notes in Physics series by Springer) represents some of the diverse lines of inquiry into aspects of strongly interacting matter under rotation.
\end{abstract}

Francesco Becattini

University of Florence

e-mail: becattini@fi.infn.it

Jinfeng Liao

Physics Department and Center for Exploration of Energy and Matter, Indiana University, $2401 \mathrm{~N}$

Milo B. Sampson Lane, Bloomington, IN 47408, USA,

e-mail:1iaoji@indiana.edu

Michael Lisa

Department of Physics, The Ohio State University, 191 West Woodruff Avenue, Columbus, OH 43210 USA.

e-mail:1isa.1@osu.edu 


\section{Milestones}

In 2005, Liang and Wang [1] predicted that spin-orbit coupling would polarize strange quarks created in non-central heavy ion collisions, resulting in emitted $\Lambda$ hyperons globally polarized along the direction of the collision angular momentum. The magnitude and momentum-dependence of the predicted polarization depended on details of specific models of quark-quark potentials, small-angle scattering approximations, and details of hadronization mechanisms.

In 2008, Becattini and collaborators [2] noted that in a hydrodynamic picture, local thermodynamic equilibrium implies a relation between the spin polarization and the rotational flow structure (vorticity). In the hydrodynamic model, vorticity can be extracted directly from the evolution, with no need to appeal to specific microscopic processes. In 2013, an equation relating the polarization of $\Lambda$ hyperons and thermal vorticity was derived [3] and such polarization was predicted to be at the level of a few percent. The first result regarding the systematic dependence of this effect on the collision beam energy, particularly in the range relevant to the beam energy scan program at the Relativistic Heavy Ion Collider (RHIC), was reported in a 2016 paper [4], providing a highly relevant insight for the later experimental measurements.

In 2017, the STAR Collaboration published [5] the first observation of global $\Lambda$ polarization from noncentral heavy ion collisions. As discussed below and throughout this Volume, most theoretical interpretations of these observations are based upon this hydrodynamic approach.

While the phenomenon of global polarization was predicted based on particleparticle interaction, the success of quantitative predictions of the hydrodynamic model to reproduce experimental observations (discussed below) seem to confirm that for spin, as for many other observables, microscopic details are less important than bulk thermodynamic properties. Below, we discuss the hydrodynamic approach to vorticity and polarization, followed by experimental observations.

We will briefly discuss the related phenomenon of vector meson spin alignment, also predicted by Liang and Wang [6] in 2005. As of now, measurements of spin alignment at the Large Hadron Collider (LHC) and RHIC are difficult to explain in any theoretical approach.

\section{Introduction}

Twenty years ago, the world's first nuclear collider began producing heavy ion collisions at energies far surpassing those previously achievable in fixed-target experiments. The goal was to produce the quark-gluon plasma (QGP)- a state of matter characterized by partonic (rather than hadronic) degrees of freedom. For decades, production and study of the QGP had long been the focus driving the field of relativistic heavy ion physics, as it holds the promise of shedding light on the non- 
perturbative region of quantum chromodynamics (QCD), the most poorly understood of the fundamental interactions in the Standard Model.

In 2005 [7, 8, 9, 10], based on a systematic and comprehensive analysis of available data, the experimental collaborations at the Relativistic Heavy Ion Collider (RHIC) confirmed that QGP is indeed created in ultra-high energy collisions. Furthermore, the data clearly indicated that the QGP was a strongly coupled fluid, contrary to some expectations that the plasma would be weakly coupled due to the combination of high temperatures and the running of the QCD coupling constant. The evidence driving this conclusion was the collective anisotropic emission distribution of hadrons from the collision- the so-called "elliptic flow." These very strong anisotropies (and the dependence upon mass and momentum) were nearly quantitatively consistent with expectations based on relativistic inviscid (ideal) hydrodynamics.

The discovery of nearly "perfect fluid" behavior had two major outcomes. Firstly, it prompted a re-evaluation of numerical QCD calculations performed on a lattice, the most reliable $a b$ initio calculations of the strong interaction. While numerically correct, lattice calculations could be misinterpreted to suggest that a weakly coupled gas of quarks and gluons was the proper paradigm for modeling collisions at RHIC. It was also realized that the QGP near the pseudo-critical transition temperature is a peculiar system: unlike ordinary matter, its microscopic interaction length is comparable to the thermal de Broglie wavelength, making the kinetic collisional description inappropriate. Nevertheless, even under such unusual conditions, the local thermodynamic equilibrium concept and hydrodynamics are still valid,. Hence, the discovery established relativistic fluid dynamics as the new paradigm for the bulk evolution of the system. Confronting increasingly sophisticated hydrodynamic calculations with data has produced valuable estimates of transport coefficients, initial parton distributions, and the QCD equation of state. Triangular and higher-order azimuthal correlations have probed the substructure of the fluid flow fields at ever finer scale.

A relativistic collision between heavy nuclei at finite impact parameter can involve angular momentum of order $10^{3 \sim 5} \hbar$. In a fluid, angular momentum can manifest as vorticity, rotational gradients of the flow and temperature fields [2]. Until recently, this aspect of the plasma had been largely ignored, as there had been no experimental observation of its effects.

In 2017, the STAR Collaboration published an observation of global hyperon polarization in $\mathrm{Au}+\mathrm{Au}$ collisions at RHIC, opening the potential to probe novel substructures of the QGP fluid at the finest possible scale. This is a rare case in which an entirely new direction is introduced to a mature field. It is especially exciting because the natural language for discussing vorticity-three-dimensional relativistic viscous hydrodynamics- has been developed to a high degree of sophistication by a large community of theorists. It is an opportunity for new insights into the physics of deconfined QCD matter, and the heavy ion community has responded with intense focus on the topic. This book represents a broad sampling of directions of inquiry into this new area of research. 


\section{Accessing subatomic vorticity}

"Lumpy" azimuthal fluid flow patterns (elliptic flow, triangular flow, etc) may be measured by azimuthal correlations between the momenta of emitted particles; this is experimentally straight-forward. Orbital angular momentum in heavy ion collisions, on the other hand, is experimentally inaccessible. Instead, one relies on coupling between the orbital ("mechanical") angular momentum of the fluid and spin of the emitted particles. The first observation of such an effect was reported more than a century ago by S. Barnett [11], in which an uncharged and un-magnetized solid metal object, when set spinning, spontaneously magnetizes 1

The analogous effect in a fluid, coupling mechanical vorticity of the bulk fluid and quantum spin polarization, was first reported by Takahashi et al, in 2016 [12]. In their experiment, liquid mercury flowing through a channel acquired local vorticity due to viscous friction with the wall. Spin-vorticity coupling produced a polarization gradient that could then be detected directly through the inverse spin Hall effect. The results could be understood by expanding angular momentum conservation in fluid dynamics, to include angular momentum transfer between the liquid and electron spin [12].

In the Barnett and Takahashi experiments, the macroscopic rotational motion was a controlled variable and the spin polarization straightfoward to measure. In highenergy nuclear collisions, the magnitude and direction of the angular momentum fluctuates from one event to the next, and a statistically significant measurement requires combining $\sim 10^{7}-10^{8}$ events. Furthermore, the particles whose polarization is to be measured are emitted at all angles at speeds approaching that of light.

These challenges are addressed by precision tracking and correlating detector subsystems in different regions of the experiment. In particular, the angular momentum in a collision is given by

$$
\boldsymbol{J}=\boldsymbol{b} \times \boldsymbol{p}_{\text {beam }},
$$

where the impact parameter, $\boldsymbol{b}$, is the transverse (to the beam direction) vector connecting the center of the target nucleus to that of the beam nucleus (where attention to the designation of beam and target is important [13]), and $\boldsymbol{p}_{\text {beam }}$ is the momentum of the beam in the collision center-of-momentum (c.o.m.) frame. The magnitude of the impact parameter, $|\boldsymbol{b}|$, is estimated by the total number of charged particles emitted roughly perpendicular to the beam in the collision c.o.m. frame, while its direction, $\hat{b}$, is estimated by the sidewards deflection of particles emitted close to the beam direction. See figure 1 for an illustration.

The flow pattern of the QGP fluid is complex and any local vorticity may fluctuate as a function of position within each droplet; however, the average vorticity must be parallel to $\boldsymbol{J}$ which is event-specific. For this reason, spin polarization projection along $\hat{J}$ is termed the "global" polarization.

\footnotetext{
${ }^{1}$ That the magnetization arose from spin polarization of the electrons was not known to Barnett and his contemporaries in 1915 , as the concept of quantum spin was not introduced until nearly a decade later.
} 

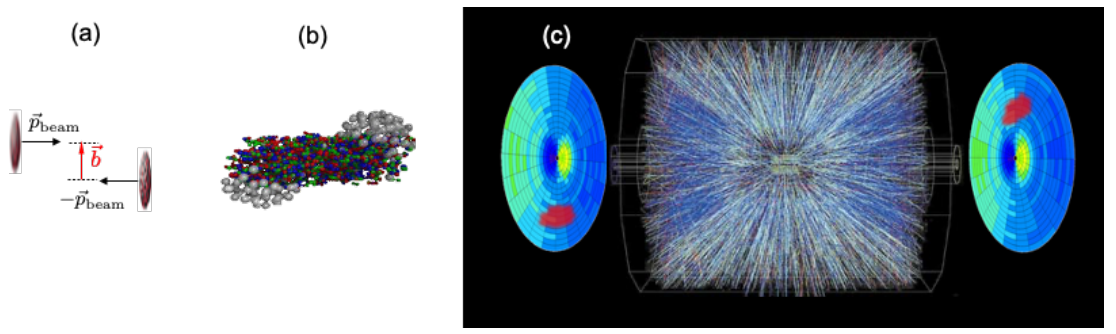

Fig. 1 The geometry of a collision. (a) Before collision: the angular momentum is determined by the impact parameter, $\boldsymbol{b}$, an uncontrolled variable that fluctuates from one collision to the next. (b) In a non-central $(|\boldsymbol{b}| \neq 0)$ collision, parts of the nuclei overlap, producing the QGP, while the so-called "spectators" continue to travel forward, experiencing only a slight impulse directed away from the collision. (c) One reconstructed event in two subsystems in STAR experiment. The Time Projection Chamber (TPC) [14] records $\sim 10^{3}$ charged particles emitted from the QGP created in the collision, while the Event Plane Detector (EPD) [15] measures spectator fragments. The magnitude and direction of $\boldsymbol{b}$ are determined, respectively, by the number of charged particles measured in the TPC and the anisotropic hit pattern in the EPD.

Having determined the direction ${ }^{2}$ of the average vorticity, the second challenge is to measure the spin polarization along that direction.

If the QGP fluid does indeed have non-vanishing vorticity, and if thermalization (complete or partial) of orbital and spin degrees of freedom does occur, then presumably all particles emitted in the collision will have their average spins aligned with $\hat{J}$. Of the zoo of particle types emitted in a heavy ion collision, the spin directions of only a few are easily measurable. In particular, particles undergoing parity-violating weak decay betray their spin direction through asymmetries in the momentum distribution of their daughters. Of this already restricted subset of particles, only a few are created in reasonable numbers to allow a significant measurement. The best candidate is the $\Lambda$ hyperon, which can be cleanly measured by its $p+\pi^{-}$decay in the TPC, as seen in panel (a) of figure 2. The decay topology is sketched in panel (b) of figure 2 An ensemble with polarization $\boldsymbol{P}_{\Lambda}$ will preferentially emit daughter protons along the direction of polarization according to

$$
\frac{d N}{d \cos \theta^{*}}=\frac{1}{2}\left(1+\alpha_{\Lambda} \boldsymbol{P}_{\Lambda} \cdot \hat{p}_{p}^{*}\right)
$$

where $\theta^{*}$ is the angle between the polarization and daughter proton momentum $\boldsymbol{p}_{p}^{*}$ in the hyperon rest frame. The decay parameter $\alpha_{\Lambda}=0.732$ determines the strength of the effect.

The global polarization is then measured by correlating information from both detector subsystems:

\footnotetext{
${ }^{2}$ In principle, the magnitude $|\boldsymbol{J}|$ of the collision's angular momentum may be estimated as well. However, not all of this angular momentum is transferred to the plasma at midrapidity [4], so usually only the direction $\hat{J}$ is of interest. This quantity is the only important ingredient to estimate vorticity in any event.
} 


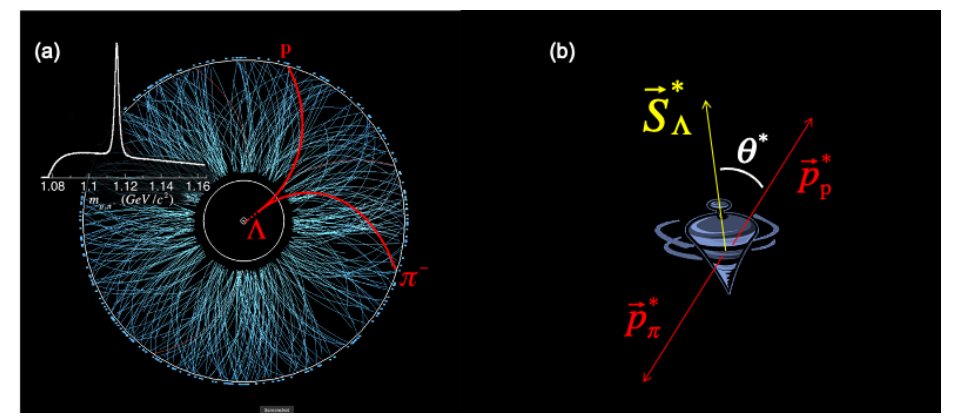

Fig. 2 (a) A $\Lambda$ hyperon detected in the STAR TPC by combining its charged proton and pion daughters. Inset: the invariant mass of daughter pairs shows a clear peak at the $\Lambda$ mass. (b) In the parity-violating decay topology, the daughter proton tends to be emitted in the direction of the parent $\Lambda$ hyperon, in the $\Lambda$ center of mass frame.

$$
\left\langle\boldsymbol{P}_{\Lambda} \cdot \hat{J}\right\rangle=\frac{8}{\pi \alpha_{H} R_{\mathrm{EP}}^{(1)}}\left\langle\sin \left(\Psi_{\mathrm{EP}, 1}-\phi_{p}^{*}\right)\right\rangle,
$$

where $\phi_{p}^{*}$ is the azimuthal angle of the daughter proton in the parent hyperon frame. In equation $3, \Psi_{E P, 1}$ is the first-order event plane angle, an estimator of the azimuthal angle of the impact parameter $b$; the resolution of this estimation is $R_{E P}^{(1)}$. Standard methods have been developed to extract both the event plane and the resolution from anisotropic particle distributions in the EPD.

The discussion thus far has described the global $\Lambda$ hyperon polarization measurement in the STAR experiment at RHIC. The ALICE experiment at the LHC performed a similar analysis, tracking charged hyperon daughters with a gas-filled TPC at midrapidity, and measuring $\Psi_{\mathrm{EP}, 1}$ with segmented detectors at forward rapidity. The STAR and ALICE measurements thus far comprise the world's dataset on global polarization, and are shown in figure 3

We offer some general remarks on figure 3 in the next section, but at the experimental level, we note that the statistical uncertainties at low $\sqrt{s_{N N}}$ are large. These uncertainties are determined by (1) the number of collision events recorded by the experiment; (2) the per-event hyperon yield; (3) the event-plane resolution $R_{\mathrm{EP}}^{(1)}$. Measurements by the STAR Collaboration in the second phase of the RHIC Beam Energy Scan (BES-II) [17] will have an order of magnitude better statistics [18] and better event plane resolution [15]; overall, the precision should increase roughly eight-fold, allowing important systematic studies [16] not currently possible.

The average "global" polarization vector must point along the direction of $\hat{J}$. On the other hand, the mean spin polarization vector for particles with specific momentum have three components which can be also measured. The component along the beam (longitudinal component) is expected to show a $2^{\text {nd }}$-order azimiuthal oscillation relative to the event plane. The amplitude and phase of this oscillation has been measured for $\mathrm{Au}+\mathrm{Au}$ collisions at $\sqrt{s_{N N}}=200 \mathrm{GeV}$ by the STAR Collaboration. Figure 4 shows the transverse momentum dependence of the $2^{\text {nd }}$ Fourier component for non-central collisions. Thanks to the excellent tracking, good event plane 


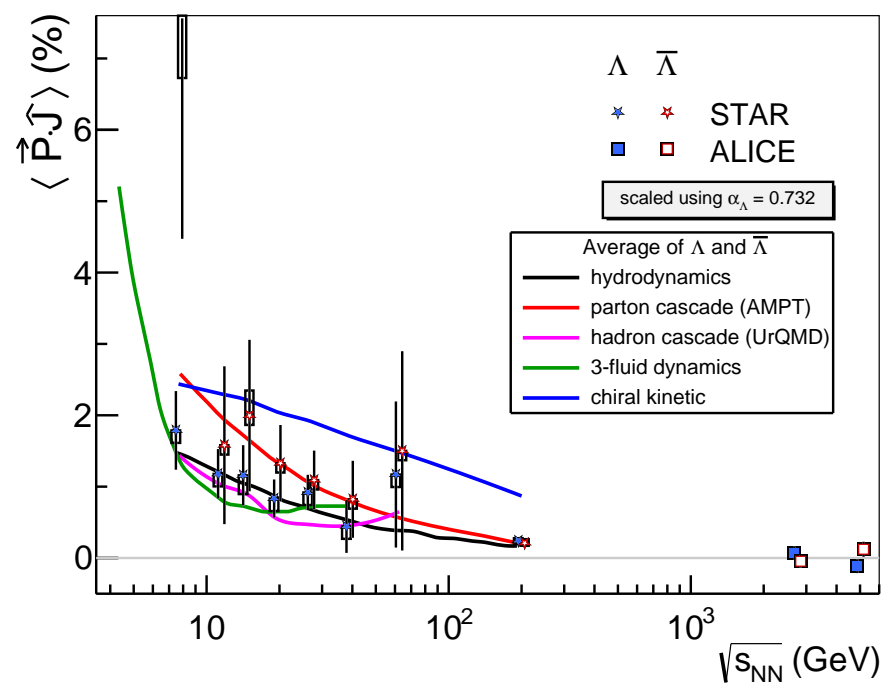

Fig. 3 The world dataset of global $\Lambda$ hyperon polarization in relativistic heavy ion collisions compared to expectations from hydrodynamic and transport simulations. Figure from [16].

resolution, and a high statistics dataset available at RHIC top energy, an oscillating sub-percent polarization signal is easily measured.

\section{From signal to physics}

\subsection{Hydrodynamics as the basis to understand hyperon polarization}

As we discussed in section 1 , the original idea for global $\Lambda$ polarization in heavy ion collisions was based on microscopic processes that drove the initial state, the transfer of angular momentum from orbital to spin degrees of freedom, and the subsequent hadronization mechanism. Assumptions and parameters were required to compute each of these components of the calculation. A detailed discussion along this line can be found in Chapter 7 [20].

The tremendous success of hydrodynamics to heavy ion physics suggests that the myriad details of microscopic processes undoubtedly at play in these complex collisions are eventually unimportant, as the system approaches local equilibrium quickly. In the earliest days of RHIC, ideal (inviscid), boost-invariant hydrodynamic calculations with simple initial conditions largely reproduced-nearly "out of the box"- the multiplicity, $p_{T}$ and mass systematics of measured elliptic flow. This success gave some confidence that equilibrium hydrodynamics was a good paradigm to understanding the collective physics of heavy ion collisions. 


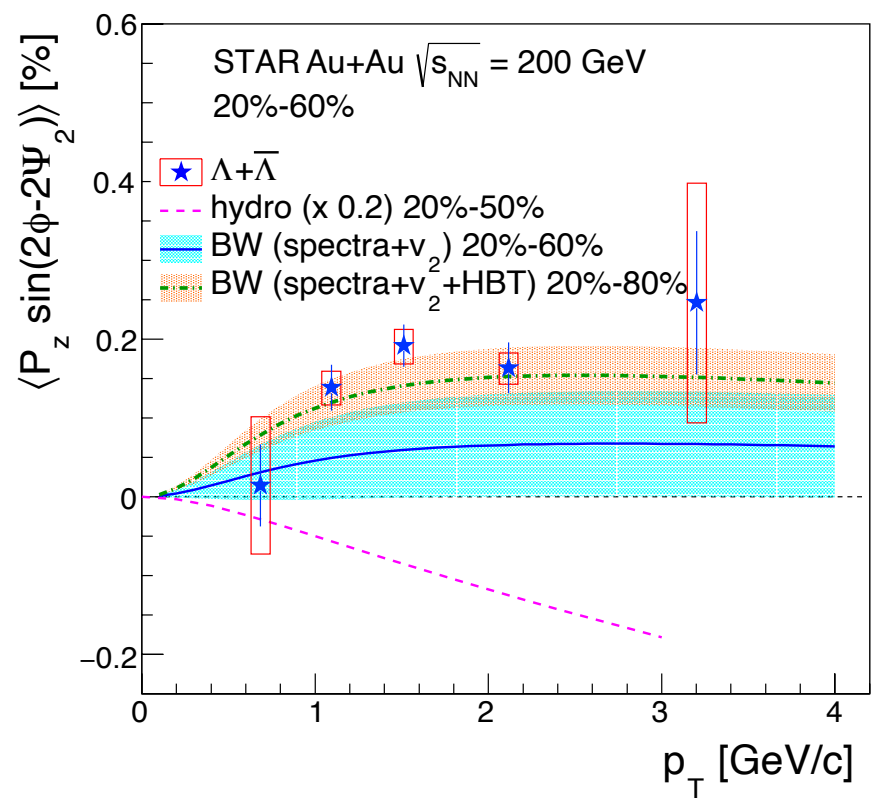

Fig. 4 The second-order Fourier coefficient of the azimuthal oscillation of the longitudinal component of the $\Lambda$ hyperon polarization. Figure from [19].

In the subsequent decades, several important insights have been achieved by working within this framework, using details in the data to probe the partonic structure of the initial state, transport coefficients, and hadronization mechanisms. These insights required considerable elaboration of the initial simple models, incorporating viscosity, baryochemical currents, vorticity, three-dimensional dynamics, and event-by-event fluctuations in the initial state. However, the close resemblance of the initial simple calculations with observations set this fruitful enterprise on firm ground.

Figure 3 suggests that the same situation exists in the study of global hyperon polarization. Theoretical curves show predictions from hydrodynamic and transport calculations, in which fluid vorticity is assumed to equilibrate with $\Lambda$ spin degrees of freedom to produce the polarization. Vorticity - more properly, thermal vorticity- 
is calculated directly from the flow field in the hydrodynamic calculations, as discussed in detail in Chapter 8 [21]. On the other hand, in the transport calculations, flow and temperature fields are calculated from the motion of multiple particles in coarse-grained spatial cells; this implicitly assumed local thermalization; see detailed discussions in Chapter 9 [22]. Eventually, in both methods, polarization of a spin $1 / 2$ fermion is obtained from the same formula relating mean spin to the thermal vorticity $\varpi$ at the leading order [3]:

$$
S^{\mu}(p)=-\frac{1}{8 m} \epsilon^{\mu \nu \rho \sigma} p_{\sigma} \frac{\int_{\Sigma} \mathrm{d} \Sigma \cdot p \varpi_{v \rho} n_{F}\left(1-n_{F}\right)}{\int_{\Sigma} \mathrm{d} \Sigma \cdot p n-F}
$$

where $S^{\mu}(p)$ is the mean spin vector and $n_{F}$ is the covariant Fermi-Dirac distribution function. The thermal vorticity is defined as the antisymmetric derivative of the fourtemperature vector field, that is:

$$
\varpi_{\mu \nu}=\frac{1}{2}\left[\partial_{\nu}\left(\frac{1}{T} u_{\mu}\right)-\partial_{\mu}\left(\frac{1}{T} u_{v}\right)\right]
$$

where $T$ and $u_{\mu}$ are local temperature field and flow velocity field, respectively. The integration in equation (4) is performed on the 3-D hadronization hypersurface $\Sigma$. The polarization vector $P^{\mu}$ is simply $S^{\mu} /|S|$ and its global, momentum integrated, value in the particle rest frame turns out to be directed along the angular momentum vector, so that, approximately one has:

$$
\langle\boldsymbol{P}\rangle \cdot \hat{J} \approx \frac{1}{2}\langle\varpi\rangle \cdot \hat{J}
$$

where the $\langle\varpi\rangle$ is the mean thermal vorticity value over the hadronization hypersurface. The above relation is a direct manifestation for the rotational polarization of microscopic spin. The theoretical underpinning of this phenomenon is to be fully elaborated through a variety of approaches such as quantum field theory (in Chapters 2 [23], 3 [24] and 4 [25]) and relativistic kinetic theory (in Chapters 5 [26] and 6 [27]).

For the most part, these models have been used to understand other observations from heavy ion collisions, and the results in figure 3 are obtained largely "out of the box". The quantitative agreement, as well as the universal decreasing trend of polarization with $\sqrt{s_{\mathrm{NN}}}$ (despite the fact that $|\boldsymbol{J}|$ increases with increasing collision energy) is a clear indication that we have at hand a paradigm to understand hyperon polarization.

That said, there are strong tensions with the existing theoretical expectations in certain observables. One is seen in figure 4 , the same hydrodynamic calculation that reproduced $\left\langle\boldsymbol{P}_{\Lambda} \cdot \hat{J}\right\rangle$ with no special tuning, predicts the wrong sign of the longitudinal polarization, $\left\langle\boldsymbol{P}_{\Lambda} \cdot \hat{z}\right\rangle$. Hence it seems that, similar to the early collective flow studies, the framework is well-grounded, while there is much to learn from the details. Chapters 8 [21], 9 [22] and 10 [28] in this Volume provide an in-depth discussion on the phenomenology study based on this framework. More broadly, the 

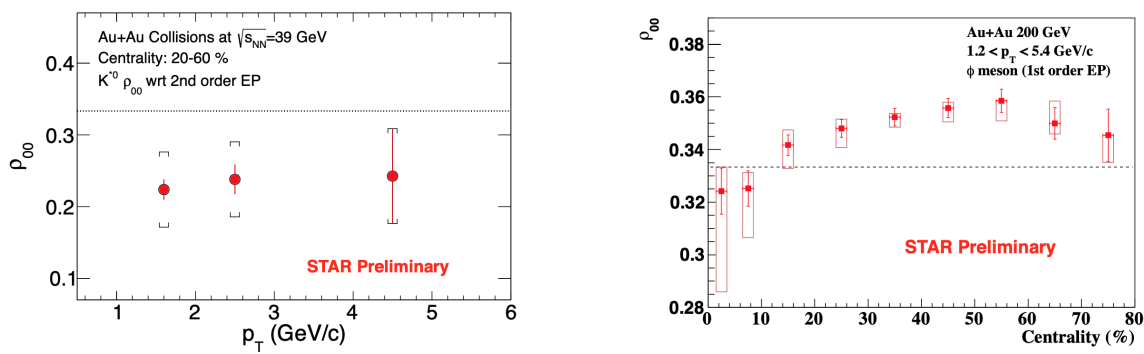

Fig. 5 Vector meson alignment in $\mathrm{Au}+\mathrm{Au}$ collisions at $\sqrt{s_{N N}}=200 \mathrm{GeV}$, measured by the STAR Collaboration at RHIC [31]. Left: $\rho_{0,0}$ for $K^{* 0}$ mesons as a function of transverse momentum for mid-central collisions. Right: $\rho_{0,0}$ for $\phi$ mesons as a function of centrality. Dashed lines indicate $\rho_{0,0}=\frac{1}{3}$, corresponding to no alignment with the normal to the event plane.

presence of global rotation has opened a new dimension for investigating its nontrivial effects, for example, on the phase structures of matter (see chapter 11 [29]) or on the interplay between orbital and spin angular momentum (c.f. chapter 12 [30]).

\subsection{Vector meson spin alignment - more complicated physics?}

In an equilibrium picture, the spins of all emitted particles will be aligned with the total angular momentum of the system. In addition to $\Lambda$ and $\bar{\Lambda}$ hyperons discussed above, preliminary results from the STAR collaboration indicate consistent polarization of $\Xi, \bar{\Xi}$ and $\Omega$ baryons [32]. Besides baryons, in principle polarization could be detected for vector mesons such as $\mathrm{K}^{*}$ or $\phi$.

The spin of a vector meson is quantified by the $3 \times 3$ spin-density matrix $\rho_{i, j}$. Becattini discusses the coupling of this quantity to fluid vorticity in Chapter 2 [23]. Due to the parity-conserving nature of their strong decay, the elements $\rho_{1,1}$ and $\rho_{-1,-1}$ cannot be separately determined. Because the trace is unity, there is only one independent diagonal element, $\rho_{0,0}$ which quantifies the component of the meson spin perpendicular to the quantization axis. As with the baryons, for the average spin, the axis of interest is $\hat{J}$, perpendicular to the event plane. Random alignment of spins would yield $\rho_{-1,-1}=\rho_{0,0}=\rho 1,1=\frac{1}{3}$. Given only experimental access to $\rho_{0,0}$, it is impossible to determine whether the meson spin is parallel or anti-parallel to $\hat{J}$, but in either case, spin alignment would imply $\rho_{0,0}<\frac{1}{3}[33$.

The 2-particle decay topology of a vector meson is related to the alignment according to [34]:

$$
\frac{\mathrm{d} N}{\mathrm{~d} \cos \theta^{*}}=\frac{3}{4}\left[1-\rho_{00}+\left(3 \rho_{00}-1\right) \cos ^{2} \theta^{*}\right]
$$

where $\theta^{*}$ is the angle between the parent spin and a daughter momentum in the parent's rest frame. At local thermodynamic equilibrium, the alignment is quadratic 


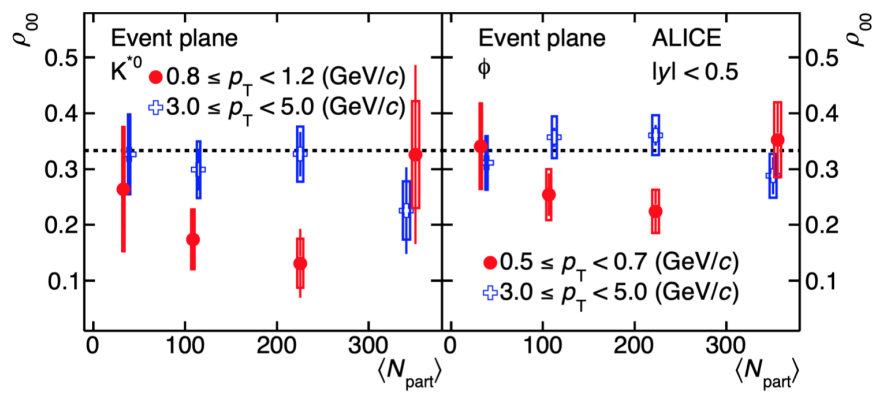

Fig. 6 Vector meson alignment, for $\mathrm{Pb}+\mathrm{Pb}$ collisions at $\sqrt{s_{N N}}=2.76 \mathrm{TeV}$, measured by the ALICE Collaboration at the LHC [36]. Left (right) panel shows $\rho_{0,0}$ for $K^{* 0}(\phi)$ mesons as a function of collision centrality, for two ranges in transverse momentum. Dashed lines indicate $\rho_{0,0}=\frac{1}{3}$, corresponding to no alignment with the normal to the event plane.

in thermal vorticity to first order [35, 16]:

$$
\frac{1}{3}-\rho_{00} \approx \frac{4}{9} \varpi^{2} .
$$

Therefore, consistency with the hyperon results would lead to the expectation $\frac{1}{3}-$ $\rho_{0,0} \approx 10^{-3}$

Experimental results deviate strongly from that expectation. Figures 5 and 6 show $K^{0 *}$ and $\phi$ alignment measurements from the STAR and ALICE experiments at RHIC and LHC, respectively. In all cases, $\left|\frac{1}{3}-\rho_{00}\right| \approx 0.1$, two orders of magnitude larger than expectations based on $P_{\Lambda}$ and vorticity considerations. Perhaps more surprisingly, STAR reports $\rho_{00}>\frac{1}{3}$ for $\phi$ mesons.

As discussed above, the hydrodynamic equilibrium ansatz seems a reliable baseline for understanding hyperon polarization, as it is for understanding much else in heavy ion physics. However, there may be many other effects at play. In their original paper [6], Liang and Wang considered different hadronization mechanisms involving polarized quarks. If vector mesons are produced by simple coalescence of a quark and antiquark with polarizations $P_{q}$ and $P_{\bar{q}}$, respectively, then

$$
\rho_{0,0}^{\text {meson }}=\frac{1-P_{q} P_{\bar{q}}}{3+P_{q} P_{\bar{q}}} \approx \frac{1}{3}-\frac{4}{9}\left(P_{q} P_{\bar{q}}\right)^{2},
$$

where the approximation holds for small polarizations. This is consistent with the hydrodynamic equilibrium prediction (equation 8 ) if $P_{q}=P_{\bar{q}}=\varpi$. It is not possible to reconcile the ALICE measurements of very small values of hyperon with large values of $\left|\frac{1}{3}-\rho_{0,0}\right|$ in a simple recombination picture [36].

Perhaps even more surprising are measurements of the STAR Collaboration at RHIC. For $K^{*}$, they report [31] values of $\frac{1}{3}-\rho_{0,0}$ similarly large as those seen at the LHC, but for $\phi$ mesons, $\rho_{0,0}>\frac{1}{3}$; c.f. figure 5 . Liang and Wang pointed out that hadronization via polarized quark fragmentation could result in $\rho_{0,0}>\frac{1}{3}$. This mechanism may be most important at large rapidity or transverse momentum, but 
could in principle play a role at midrapidity, where these measurements are made. However, naively, if fragmentation is the dominant hadronization mechanism, $K^{*}$ and $\phi$ should be affected similarly. Furthermore, it would seem natural that quark hadronization would be more important at LHC energies than at RHIC.

Sheng, Olivia and Wang [37] propose that an entirely new physical effect could be at play, in which a hypothetical mean $\phi$ field couples to the system angular momentum. Depending on the values of several parameters, $\rho_{0,0}^{\phi}$ could be greater or less than $\frac{1}{3}$. In principle, by fine-tuning [37] the energy dependence of four parameters, this model might accommodate $\rho_{0,0}^{\phi}>\frac{1}{3}$ at RHIC energies and $\rho_{0,0}^{\phi}<\frac{1}{3}$ at the LHC. Because this model is not expected to apply to $K^{* 0}$ mesons [37], it will be important to identify independent measurements that can constrain and verify its assumptions.

In summary, it is clear that the situation with spin alignment of vector mesons is very different than that for hyperon polarization. In the latter case, the equilibrium hydrodynamic paradigm which works well for other aspects of heavy ion collisions seems a reasonable starting point; polarization then allows a more sensitive probe of the system evolution at the finest scales. For the vector mesons, however, it is clear that observations cannot be explained by this established paradigm. Competing effects from multiple hadronization mechanisms, hadronic effects, and novel mean fields may be at play, differentially affecting the different particle species and different collision energies. See Chapter 7 [20] by Gao, et al in this Volume, for an extensive discussion. The phenomenon of vector meson spin alignment deserves continued intense theoretical focus; at the moment, the situation is too unclear to summarize what might be learned.

\subsection{Future experimental work}

Among the most pressing issues in the field of heavy ion physics is the existence and consequences of an intense, long-lived magnetic field. Its presence could allow experimental access to novel effects due to chiral symmetry restoration. Because $\Lambda$ and $\bar{\Lambda}$ have opposite magnetic moments, a strong $B$-field at hadronization would lead to a polarization "splitting" [35, 38, 39]. The magnitude of the splitting remains below the statistical sensitivity of existing measurements, but the ongoing BES-II campaign at RHIC is expected to either discover the splitting or set meaningful limits on possible magnetic effects.

While the average ("global") polarization must align with the total angular momentum of the collision, hydrodynamic and transport simulations predict a rich flow structure featuring nontrivial local vorticity. The longitudinal polarization results in figure 4 represent the first observation of such an effect. However, more complicated effects may be present on an event-by-event basis, leading to vorticity "hot spots" that may be revealed by spin-spin correlations [40]. Experimental searches for such a signal are ongoing at RHIC, but two-particle tracking artifacts make them highly challenging. 
As discussed above, the physics driving vector meson spin alignment is apparently much more complicated than that behind $\Lambda$ polarization. The STAR Collaboration at RHIC has presented a nearly finalized study [32] of polarization of $\Xi$ and $\Omega$ hyperons which appear consistent with the $\Lambda$ polarizations, with small massdependent effects.

While the total system angular momentum decreases with reduced collision energy, the largest global polarization is observed at the lowest energy. It will be important to measure polarization at still lower energies, below the energy threshold for QGP formation and at energy densities below the limits of applicability of hydrodynamics. The BES-II program at RHIC includes a fixed-target campaign already producing results [41] in this regime. Much higher statistics datasets at low energy are expected at the NICA and FAIR facilities soon to commence operation.

The energy dependence of the polarization signal may reflect an evolution of rotational flow structure away from midrapidity, where measurements have focused thus far, as the collision energy increases. Experiments with good tracking near beam rapidity may probe strong vorticity resulting from the breakdown of longitudinal boost-invariance, challenging hydrodynamic and transport simulations more stringently than possible previously.

\section{Summary and Outlook}

Over several decades, the field of relativistic heavy ion physics has matured and focused on the creation and study of the quark-gluon plasma. Hydrodynamics and transport theory have provided a useful paradigm in which to interpret a wide diversity of experimental results from high-energy collisions at RHIC and the LHC. Theory and models based on this paradigm have become increasingly sophisticated, simulating the entire evolution of the dynamic system and making quantitative connection to the initial state and fundamental transport coefficients.

The observation of rotational phenomena has opened an exciting new direction into this well-developed and fertile environment, a rare example of a truly new development in a mature field. First measurements of global hyperon polarization are largely consistent with predictions from existing hydrodynamic and transport simulations, indicating that the tools are at hand, to understand the phenomenon. More differential measurements, of the azimuthal dependence of global and longitudinal hyperon polarization, are more difficult to understand; the effects have magnitudes in line with standard expectations, but reproducing the sign of the observed oscillations may require nontrivial revisions to our current understanding. On the other hand, vector meson spin alignment- presumably related to hyperon polarizationis quantitatively and qualitatively impossible to understand solely in terms of the hydrodynamic paradigm that successfully explains other observables; here, there may be numerous competing effects that depend nontrivially on particle species and collision energy, including a newly-proposed coherent mesonic mean field. 
Thus, it appears that the new phenomena of strongly interacting QCD matter under rotation may be addressed by current theory and models, while at the same time requiring new insights. The contributions to this book represent a broad sample of some of the early theoretical efforts- from fundamental theory to phenomenologyto determine the physics behind these phenomena. New insights are bound to result from continued theoretical focus and upcoming experimental results. The following pages are the first chapters in what will surely be a much longer story.

\section{Acknowledgements}

This work is supported in part by U.S. Department of Energy grant DE-SC0020651 and by U.S. National Science Foundation grant PHY-1913729.

\section{References}

1. Z.T. Liang, X.N. Wang, Phys. Rev. Lett. 94, 102301 (2005). DOI 10.1103/PhysRevLett.94. 102301. [Erratum: Phys.Rev.Lett. 96, 039901 (2006)]

2. F. Becattini, F. Piccinini, J. Rizzo, Phys. Rev. C 77, 024906 (2008). DOI 10.1103/PhysRevC. 77.024906

3. F. Becattini, V. Chandra, L. Del Zanna, E. Grossi, Annals Phys. 338, 32 (2013). DOI 10.1016/ j.aop.2013.07.004

4. Y. Jiang, Z.W. Lin, J. Liao, Phys. Rev. C94(4), 044910 (2016). DOI 10.1103/PhysRevC.94. 044910,10.1103/PhysRevC.95.049904. [Erratum: Phys. Rev.C95,no.4,049904(2017)]

5. L. Adamczyk, et al., Nature 548, 62 (2017). DOI 10.1038/nature23004

6. Z.T. Liang, X.N. Wang, Phys. Lett. B 629, 20 (2005). DOI 10.1016/j.physletb.2005.09.060

7. J. Adams, et al., Nucl. Phys. A 757, 102 (2005). DOI 10.1016/j.nuclphysa.2005.03.085

8. K. Adcox, et al., Nucl. Phys. A 757, 184 (2005). DOI 10.1016/j.nuclphysa.2005.03.086

9. B. Back, et al., Nucl. Phys. A 757, 28 (2005). DOI 10.1016/j.nuclphysa.2005.03.084

10. I. Arsene, et al., Nucl. Phys. A 757, 1 (2005). DOI 10.1016/j.nuclphysa.2005.02.130

11. S.J. Barnett, Phys. Rev. 6, 239 (1915). DOI 10.1103/PhysRev.6.239. URL https://link. aps.org/doi/10.1103/PhysRev.6.239

12. M. Takahashi, et al., Nature Phys. 12, 52 (2016). DOI 10.1038/nphys3526

13. B. Abelev, et al., Phys. Rev. C 95, 039906 (2017). DOI 10.1103/PhysRevC.95.039906

14. M. Anderson, et al., Nucl. Instrum. Meth. A499, 659 (2003). DOI 10.1016/S0168-9002(02) 01964-2

15. J. Adams, et al., Nucl. Instrum. Meth. A 968, 163970 (2020). DOI 10.1016/j.nima.2020. 163970

16. F. Becattini, M.A. Lisa, Ann. Rev. Nucl. Part. Sci. 70, 395 (2020). DOI 10.1146/ annurev-nucl-021920-095245

17. A. Bzdak, S. Esumi, V. Koch, J. Liao, M. Stephanov, N. Xu, Phys. Rept. 853, 1 (2020). DOI 10.1016/j.physrep.2020.01.005

18. M. Aggarwal, et al., arXiv:1007.2613 (2010)

19. J. Adam, et al., Phys. Rev. Lett. 123(13), 132301 (2019). DOI 10.1103/PhysRevLett.123. 132301

20. J.H. Gao, Z.T. Liang, Q. Wang, X.N. Wang, arXiv:2009.04803 (2020)

21. I. Karpenko, arXiv:2101.04963 (2021)

22. X.G. Huang, J. Liao, Q. Wang, X.L. Xia, arXiv:2010.08937(2020) 
23. F. Becattini, arXiv:2004.04050(2020)

24. M. Buzzegoli, arXiv:2011.09974 (2020)

25. V.E. Ambrus, E. Winstanley, arXiv:1908.10244 (2019)

26. L. Tinti, W. Florkowski, arXiv:2007.04029(2020)

27. Y. Jiang, X. Guo, P. Zhuang, this Volume (2021)

28. G. Cao, I. Karpenko, this Volume (2021)

29. H.L. Chen, X.G. Huang, J. Liao, this Volume (2021)

30. K. Fukushima, S. Pu, arXiv:2001.00359 (2020)

31. C. Zhou, Nucl. Phys. A 982, 559 (2019). DOI 10.1016/j.nuclphysa.2018.09.009

32. T. Niida, Presentation at RHIC Users Meeting (2020)

33. E. Leader, Spin in particle physics, vol. 15 (Cambridge University Press, 2011)

34. K. Schilling, P. Seyboth, G.E. Wolf, Nucl. Phys. B 15, 397 (1970). DOI 10.1016/ 0550-3213(70)90070-2. [Erratum: Nucl.Phys.B 18, 332 (1970)]

35. F. Becattini, I. Karpenko, M. Lisa, I. Upsal, S. Voloshin, Phys. Rev. C 95(5), 054902 (2017). DOI 10.1103/PhysRevC.95.054902

36. S. Acharya, et al., Phys. Rev. Lett. 125(1), 012301 (2020). DOI 10.1103/PhysRevLett.125. 012301

37. X.L. Sheng, L. Oliva, Q. Wang, Phys. Rev. D 101(9), 096005 (2020). DOI 10.1103/PhysRevD. 101.096005

38. B. Müller, A. Schäfer, Phys. Rev. D 98(7), 071902 (2018). DOI 10.1103/PhysRevD.98.071902

39. X. Guo, J. Liao, E. Wang, Sci. Rep. 10(1), 2196 (2020). DOI 10.1038/s41598-020-59129-6

40. L.G. Pang, H. Petersen, Q. Wang, X.N. Wang, Phys. Rev. Lett. 117(19), 192301 (2016). DOI 10.1103/PhysRevLett.117.192301

41. J. Adam, et al., arXiv:2007.14005 (2020) 